\title{
ON THE PARTITIONS OF A GROUP AND THE RESULTING CLASSIFICATION*
}

\author{
BY J. W. YOUNG
}

1. Introduction. I propose the following definition: A partition of any group $G$ is any class [H] of subgroups of $G$ such that every element other than the identity of $G$ is contained in one and only one $H$. The concept thus defined arose very naturally in connection with the problem of defining a general algebra without introducing the idea of a field. $\dagger$ After this paper was written Professor G. A. Miller called my attention to the fact that he had considered the partitions of a group (though not under the present terminology) in a paper presented to the Society in 1906. His methods and point of view are, however, entirely different and only one of his theorems duplicates a result of the present paper.

Even though the results of the present paper find their immediate application in the domain of general algebras, it seemed desirable to publish them in a separate paper. The concept itself and the process defined in $\$ 2$ below for the determination of the so-called primitive partition of a group would appear to be of possible value in the general theory of groups and the resulting classification of all groups into types would seem to possess elements of interest. This is due especially to the fact that results in what may be

* Presented to the Society, September 9, 1926.

† See a paper (not yet published), On the definition of the Scorza-Dickson algebras, presented to the Society at New York, February 26, 1927; abstract, this Bulletin, vol. 33 (1927), p. 265. The linear systems of order one in an algebra as defined in Dickson, Algebras and their Arithmetics (University of Chicago Press, 1923), pp. 9, 10, form a partition of the addition group of the algebra.

$\ddagger$ G. A. Miller, Groups in which all the operators are contained in a series of subgroups such that any two have only identity in common, this Bulletin, vol. 12 (1905-06), p. 446. 
called the general theory of groups are comparatively rare, while the literature of special classes of groups (finite, continuous, etc.) is enormous. In what follows we place no restrictions on the group $G$ beyond what is implied in the definition of any group.*

2. The Classification of Groups into Types. The Primitive Partition. Let $G_{1, s}$ be the cyclical subgroup of $G$ generated by the element $s(\neq 1)$ of $G . G_{1, s}$ then consists of all the positive and negative integral powers of $s$ and $1=s^{0}$. If there exists a finite integer $m>1$ such that $s^{m}=1, s$ is of finite order and the smallest such integer is called the order of $s$. Let $G_{1,(s)}$. be any cyclical subgroup of $G$ containing $s$. Let $G_{2, s}=\left\{\left[G_{1,(s)}\right]\right\}$ be the subgroup of $G$ generated by all the groups $G_{1, t}$ containing $s$, i. e., the smallest subgroup of $G$ containing all the $G_{1,(s)}$. Evidently $G_{2, s}$ is uniquely determined by $s$. In general let $G_{k+1, s}$ be the subgroup of $G$ generated by all the $G_{k, t}$ containing $s(k=1,2, \cdots)$. Let $\left[G_{k}\right]$ be the class of all subgroups $\left[G_{k, s}\right]$ as $s$ ranges over $G$.

We thus obtain for every $s$ of $G$ a sequence of subgroups $G_{1, s}, G_{2, s}, \cdots$ such that every $G_{k, s}(s \neq 1, k>1)$ contains the preceding $G_{k-1, s}$ :

$$
G_{1, s} \leqq G_{2, s} \leqq \cdots \leqq G_{k, s} \leqq \cdots .
$$

We also obtain a sequence of classes of subgroups $\left[G_{1}\right]$, $\left[G_{2}\right], \cdots$, such that every element of $G$ is contained in one or more subgroups $G_{k}$ for every value of $k=1,2, \cdots$, and such that every $\left[G_{k}\right](k>1)$ contains the preceding class $\left[G_{k-1}\right]$ in the sense that every $G_{k-1, s}$ is contained in $G_{k, s}$. We indicate this by writing $\left[G_{k-1}\right] \leqq\left[G_{k}\right] ;\left[G_{k-1}\right]=\left[G_{k}\right]$, if and only if, for every $s$ we have $G_{k-1, s}=G_{k, s}$. With this notation we then have

* A class of elements, $s, t, \cdots$, is said to form a group with respect to a law of combination (indicated by juxtaposition) if the following conditions are satisfied: (1) the product $s t$ shall be a uniquely defined element of $G$ for every ordered pair $s, t$ of $G$; (2) for every three elements of $G$, (st) $u=s(t u)$; (3) there exists in $G$ an element 1 , called the identity, such that for every $s$ of $G, s 1=1 s=s$; (4) for every $s$ of $G$ there exists an inverse $s^{\prime}$ such that $s s^{\prime}=1$. 


$$
\left[G_{1}\right] \leqq\left[G_{2}\right] \leqq \cdots \leqq\left[G_{k}\right] \leqq \cdots .
$$

If there exists a finite integer $n$ such that for every $s$ of $G$

$$
G_{n, s}=G_{n+1, s}=\left\{\left[G_{n,(s)}\right]\right\},
$$

$G_{n,(s)}$ must consist of the single group $G_{n, s}$, i. e., every $(s \neq 1)$ of $G$ is in one and only one $G_{n, s}$; or, if $s$ and $t$ are any two elements of $G(s \neq 1, t \neq 1)$, then either $G_{n, s}=G_{n, t}$ or $G_{n, s}$ and $G_{n, t}$ have only the identity in common. Hence we may state the following theorem.

THEOREM 1. If there exists an $n$ such that $\left[G_{n}\right]=\left[G_{n \pm 1}\right]$, then two distinct $G_{n}$ 's have only the identity in common; and conversely.

To prove the converse, we observe that if two distinct $G_{n}$ 's have only the identity in common and $s(\neq 1)$ is any element of $G,\left[G_{n,(s)}\right]$ consists of the single group $G_{n, s}$.

Now let $n$ be the smallest integer for which $\left[G_{n}\right]=\left[G_{n+1}\right]$. Our sequence $\left[G_{k}\right]$ then becomes

$$
\left[G_{1}\right]<\left[G_{2}\right]<\cdots<\left[G_{k}\right]=\left[G_{k+1}\right]=\cdots ;
$$

and for every $s$ there exists a smallest $n_{s}$ such that

$$
G_{1, s}<G_{2, s}<\cdots<G_{n_{s}, s}=G_{n_{s}+1, s}=\cdots .
$$

Clearly we have $n_{s} \leqq n$, for every $s$.

Under this hypothesis our sequence $\left[G_{k}\right]$ terminates, in a sense, with $\left[G_{n}\right]$ and this class is a partition of $G$.

If, on the other hand, there exists no finite $n$ such that $\left[G_{n}\right]=\left[G_{n+1}\right]$, there must be in $G$ an $s$ such that $G_{1, s}<G_{2, s}<$ $\ldots<G_{k, s}<\ldots, k \doteq 1,2, \ldots, G$ must then contain a smallest subgroup $G_{\omega, s}$ containing all the $G_{k, s}$ for all finite values of $k . G_{\omega+1, s}$ is then defined as the group generated by all the $G_{\omega, t}$ containing $s$, and the process previously defined leads to sequences of groups

$$
G_{\omega, s} \leqq G_{\omega+1, s} \leqq \cdots \leqq G_{2 \omega, s} \leqq \cdots \leqq G_{\omega^{2}, s} \leqq \cdots,
$$

and to sequences of classes of groups

$$
\left[G_{\omega}\right] \leqq\left[G_{\omega+1}\right] \leqq \cdots \leqq\left[G_{2 \omega}\right] \leqq \cdots \leqq\left[G_{\omega^{2}}\right] \leqq \cdots .
$$


If $N$ be the smallest integer, finite or transfinite, for which $\left[G_{N}\right]=\left[G_{N+1}\right]$, the group $G$ is said to be of type $N$.* $^{*}$ Theorem 1 and the results obtained since may now be stated as follows.

THEOREM 2. If $G$ is a group of type $N$ two distinct $G_{N}$ 's have only the identity in common, and conversely. The class $\left[G_{N}\right]$ is then a partition of $G$.

In a group of type $N$ the partition $\left[G_{N}\right]$ is evidently uniquely determined. It may be called the primitive partition of $G$. The primitive partition may, of course, consist simply of $G$ itself. A partition containing more than one group may be called a proper partition. It is evident from the way in which the primitive partition of a group is obtained, that unless the primitive partition is proper, a group cannot have any proper partition. In fact, the following theorem is obvious:

THEOREM 3. If $[H]$ is any partition of a group $G$ of type $N$ and $\left[G_{N}\right]$ is the primitive partition of $G$, then for every $s$ in $G$ we have $G_{N, s} \leqq H_{s} . \dagger$

Indeed, we have $H_{s}=\left\{\left[G_{N, t}\right]\right\}$, as $t$ ranges over $H_{s}$.

3. The Partitions of an Abelian Group. If an infinite abelian group $G$ contains elements of finite order, the latter form a subgroup $F$, invariant in $G$. The quotient group $G / F$ then contains no elements of finite order, and $G$ may be considered as the direct product of $F$ and $G / F$.

We consider first an abelian group none of whose elements is of finite order.

Theorem 4. Any two cyclical subgroups $G_{1,8}$ and $G_{1, t}$ of an abelian group $G$ with no elements of finite order which have a common element $(\neq 1)$ are contained in a cyclical subgroup $G_{1, u}$ of $G$.

* This classification for larger values of $N$ is purely formal. I do not know whether groups of type $N$ exist even for all finite values of $N$.

$\dagger H_{s}$ denotes the $H$ containing $s$. 
Let $s \neq t$, and let $s^{m}=t^{n}(\neq 1)$ be the common element. If either $m$ or $n$ equals 1 , the theorem is proved. Moreover $m$ and $n$ cannot be equal; for, the relation $s^{m}=t^{m}$ would imply $\left(s^{-1} t\right)^{m}=1$ and, since $s^{-1} t \neq 1$, this would imply that $G$ contains an element of finite order. We may assume that $m$ and $n$ are prime to each other; for, the relations $m=k m^{\prime}$, $n=k n^{\prime}(k>1)$, would imply $\left(s^{-m^{\prime}} t^{n^{\prime}}\right)^{k}=1$, which leads to $s^{m^{\prime}}=t^{n^{\prime}}$. Two integers, $x, y$, therefore, exist such that $m x+n y=1$. Now, let $t^{x} s^{y}=u$. We then have

$$
\begin{aligned}
& s=s^{m x} s^{n y}=t^{n x} s^{n y}=u^{n} ; \\
& t=t^{m x} t^{n x}=t^{m x} s^{m y}=u^{m} .
\end{aligned}
$$

This proves the theorem.

As an immediate corollary we observe that if any finite number of cyclical subgroups of an abelian group $G$ with no elements of finite order have an element $(\neq 1)$ in common, there exists a cyclical subgroup of $G$ which contains them all. We may now prove the following theorem.

THEOREM 5. In any abelian group $G$ contains no elements of finite order, $\left[G_{2}\right]$ is a partition of $G$.

To prove the theorem it is sufficient to show that if two subgroups $G_{2, s}$ and $G_{2, t}$ have an element $c(\neq 1)$ in common they coincide. $G_{2, s}$ is the smallest group containing all the $G_{1,(s)}$. Since $c$ is in $G_{2, s}, c$ is the product of a finite number of elements each of which is an element of some $G_{1,(s)}$. By the corollary of Theorem 4 , this set of $G_{1,(s)}$ are all contained in a $G_{1,(s)}$. In other words, there exists a $G_{1, p}$ containing both $c$ and $s$. Similarly, there exists a $G_{1, q}$ containing both $c$ and $t$. Let $t_{1}(\neq 1)$ be any element of $G_{2, t}$. Reasoning as before proves the existence of a $G_{1, u}$ containing $t, c$, and $t_{1}$. The groups $G_{1, p}$ and $G_{1, u}$, have $c$ in common $(c \neq 1)$; there exists, therefore, a $G_{1, v}$ containing $t, c, t_{1}$, and $s . G_{1, v}$ is, therefore, a $G_{1,(s)}$ and hence $t_{1}$ is contained in $G_{2, s}$. Every element of $G_{2, t}$ is then an element of $G_{2,8}$. Similarly, it follows that every element of $G_{2,8}$ is an element of $G_{2, t}$. 
Corollary. Any abelian group containing no elements of finite order is of type $\leqq 2$.

Now, let $G$ be any abelian group all of whose elements are of finite order. Let $G$ contain an element $s_{1}$ of order $p q$ where $p$ and $q$ are prime numbers (not excluding the possibility $p=q$ ); let $s_{1}^{q}=s$, whence $s^{p}=1$; and let $t$, of order $m$, be any other element of $G$.

We consider, first, the case where $m$ contains a prime factor different from $p$. Let $p^{\alpha}$ be the highest power of $p$ contained in $m$, so that $m=p^{\alpha} m^{\prime}$, where $m^{\prime}(>1)$ is prime to $p$. Then $t^{p^{\alpha}}=t_{1}$ is of order $m^{\prime}$, and there exists an $n$ such that $m^{\prime} n \equiv 1(\bmod p)$. Then,

$$
\left(s_{1} t_{1}\right)^{q m^{\prime} n}=\left(s_{1}^{q}\right)^{m^{\prime} n}=s^{m^{\prime} n}=s ;
$$

and, hence, $G_{1, s_{1}}$ and $G_{1, s_{1} t_{1}}$ contain $s$. Therefore,

$G_{2, s} \quad$ contains $s_{1}$ and $s_{1} t_{1}$ and, hence, $s_{1}$ and $t_{1}$;

$G_{2, t} \quad$ contains $t_{1}$;

$G_{3, t_{1}} \quad$ contains $s$ and $t$;

and $G_{4,8}$ contains $t$.

We consider, next, the case where $m=p^{\alpha}, \alpha>1$. Let $t^{p}=t_{1} ;$ then, $(s t)^{p}=t_{1} ;$ and

$G_{1, t} \quad$ contains $t_{1}$;

$G_{1, s t}$ contains $t_{1}$;

$G_{2, t_{1}}$ contains $t$ and $s t$, and, hence, $s$;

and

$$
G_{3, s} \quad \text { contains } t \text {. }
$$

We consider, finally, the case $m=p$. Let $s_{1}^{p}=s^{\prime}$. Then

$$
\begin{array}{ll}
G_{1, s_{1}} & \text { contains } s^{\prime} ; \\
G_{1, s_{1} t} & \text { contains } s^{\prime} ; \\
G_{2, s^{\prime}} & \text { contains } s_{1} \text { and } s_{1} t, \text { and hence, } t .
\end{array}
$$

Hence, in every case, if $G$ contains an element of order $p q$, there exists an element $s$ (called $s^{\prime}$ in the last case), such that $G_{4, s}=G$. Hence no group $G$ containing an element of order $p q$ has a proper partition. If, however, all the elements of $G$ are of the same prime order $p$, the cyclical subgroups of $G$ evidently form a partition. Hence, we have the following theorem. 
THEOREM 6. If an abelian group all of whose elements are of finite order has a proper partition all of its elements are of the same prime order $p$; and every abelian group containing more than $p$ elements and all of whose elements are of the same prime order $p$ has a proper partition $\left[G_{1}\right]$.

Corollary 1. An abelian group of finite order has a proper partition if, and only if, it is of order $p^{m}(m>1)$ and of type $(1,1, \cdots, 1)$.*

Corollary 2. An abelian group all of whose elements are of finite order is of type $\leqq 4$.

Now let $G$ be an abelian group containing elements of finite order and also elements of infinite order. $G$ is then the direct product of a group $F$ containing only elements of finite order and a group $I$ containing no elements of finite order. Let $s$ be any element of $I$ and let $t$, of order $m$, be any element of $F$. Then we have $(s t)^{m}=s^{m}$, so that $G_{1, s t}$ and $G_{1, s}$ have $s^{m}$ in common. $G_{2, s^{m}}$ then contains $s$ and $s t$, and hence $s$ and $t$. Therefore, $G_{3, s}$ contains $t$; i. e., $G_{3,8}$ contains $F$. The class of all $\left[G_{3, s}\right]$ as $s$ ranges over $I$ all have $F$ in common, therefore, and hence any $G_{4, t}$, where $t$ is in $F$, contains all the elements of $I$ and also of $F$. Hence we have $G_{4, t}=G$. We have, therefore, the following theorem.

THEOREM 7. An abelian group containing elements of finite and also of infinite order has no proper partition.

In connection with previous results we have the following result.

Corollary. Every abelian group is of type $\leqq 4$.

We may combine the results of Theorems 5,6 , and 7 as follows.

THEOREM 8. If an abelian group has a proper partition, either it contains no elements of finite order or all its elements are of the same prime order. In any such group $G$, the class of subgroups $\left[G_{2}\right]$ is a partition.

* This corollary was proved by G. A. Miller, loc. cit., p. 448. 
4. L-Partitions. In connection with the contemplated application to general algebras and possibly in other connections the following concept is important. Let $[H]$ be any partition of a group $G$ and let $K=\left\{H_{s_{1}}, H_{s_{2}}, \cdots, H_{s_{k}}\right\}$ be the group generated by any finite number of $H$ 's. If any $H_{p}$ having an element $(\neq 1)$ in common with any $K$ is entirely contained in $K$, the partition $[H]$ is called an $L$-partition.

We first observe the following theorem.

THEOREM 9. The primitive partition of an abelian group $G$ all of whose elements are of the same prime order $p$ is an $L$-partition.

For, the partition consists of the cyclical subgroups $\left[G_{1}\right]$ of order $p$. If $\left\{G_{1, s_{1}}, G_{1, s_{2}}, \cdots, G_{1, s_{k}}\right\}$ contains an element $t$ of a cyclical subgroup we may take this subgroup to be $G_{1, t}$, and we should have $t=s_{1}{ }^{\alpha_{1}} s_{2}{ }^{\alpha_{2}} \cdots s_{k}{ }^{\alpha_{k}}$. But all the powers of $t$ are then contained in $\left\{G_{1, s_{1}}, G_{1, s_{2}}, \cdots, G_{1, s_{k}}\right\}$.

We seek next the condition that the primitive partition of an abelian group $G$ containing no elements of finite order be an $L$-partition. Let $[H]$ be a proper partition of $G$, let $s(\neq 1)$ be any element of $G$ and let $t(\neq 1)$ be any element of $G$ not in $H_{s}$. Let $s^{-1} t^{k}=u$ ( $k$ any integer) and consider the group $\left\{H_{s}, H_{u}\right\}$. Clearly $H_{s} \neq H_{u}$; for, the assumption that $u$ is in $H_{s}$ would imply $H_{s}=H_{t}$. Since $\left\{H_{s}, H_{u}\right\}$ has $t^{k}=s u$ in common with $H_{t}$, if $[H]$ is an $L$-partition $\left\{H_{s}, H_{u}\right\}$ must contain $t$. Hence, there must exist elements $s_{1}, u_{1}$ of $H_{s}$ and $H_{u}$, respectively, such that $t=s_{1} u_{1}$. This gives $t^{k}=s_{1}{ }^{k} u_{1}{ }^{k}=s u$, whence we have $s_{1}{ }^{k} s^{-1} \cdot u^{k} u^{-1}=1$. Since $H_{8}$ and $H_{u}$ have only identity in common, this gives $s_{1}{ }^{k}=s$. Since $s$ was any element in $G$ and $k$ was any integer, it follows that, if $G$ admits an $L$-partition, every element of $G$ must have a kth root in $G$ for every integer $k$.

We now prove that this necessary condition is also sufficient in order that a proper primitive partition of $G$ be an $L$-partition. Let now $[H]$ be a proper primitive partition of $G$, and let $K=\left\{H_{s_{1}}, H_{s_{2}}, \cdots, H_{s_{k}}\right\}$ be the subgroup 
generated by any finite number of $H^{\prime}$ s. Let $t^{\prime}(\neq 1)$ be an element common to $K$ and any other $H_{t}$, so that we have $t^{\prime}=s_{1}^{\prime} s_{2}^{\prime} \cdots s_{k}^{\prime}$, where $s_{i}^{\prime}$ is in $H_{s i}$. Let $t$ be any other element of $H_{t}$. Then, by Theorem 5, there exists an element $u$ of $H_{t}$ and integers $m$ and $n$ such that $t=u^{m}$ and $t^{\prime}=u^{n}$. Under the condition imposed on $G$ there exist in $G$ elements $v_{i}$, such that $v_{i}^{n}=s_{i}^{\prime}$, for every $i$ from 1 to $k$, and each of these $v_{i}$ are, of course, in $H_{s_{i}}$. We have, then,

$$
t^{\prime}=s_{1}^{\prime} s_{2}^{\prime} \cdots s_{k}^{\prime}=\left(v_{1} v_{2} \cdots v_{k}\right)^{n}=u^{n} .
$$

But in a group $G$ containing no elements of finite order the last equality implies $u=v_{1} v_{2} \cdots v_{k}$. Hence, $u$ is in $K$ and, hence, $t$ is in $K$. We have, therefore

THEOREM 10. The necessary and sufficient condition that a proper primitive partition of an abelian group $G$ containing no elements of finite order be an L-partition is that every element of $G$ have a kth root in $G$ for every integer $k$.

Dartmouth College

\section{ANALYTIC FUNCTIONS WITH ASSIGNED VALUES*}

BY PHILIP FRANKLIN

1. Introduction. The question of determining when the values of a function at a denumerably infinite set of points in a finite region determine a function analytic in this region, and if so the power series for the function in question, has recently been raised by Professor T. H. Hildebrandt. $\dagger$ It is well known that if the function in question exists, it is uniquely determined. $\ddagger$ The usual proof gives a process for determining the coefficients in the power series, in which

* Presented to the Society, February 26, 1927.

$\dagger$ This Bulletin, vol. 32 (1926), p. 552.

$\ddagger$ W. F. Osgood, Funktionentheorie, vol. 1 (1912), p. 337. 